\title{
Comprensión lectora mediante la investigación como estrategia pedagógica apoyada en TIC
}

\section{Reading comprehension through research as a pedagogical strategy supported by TIC}

DOI: http://dx.doi.org/10.17981/cultedusoc.9.3.2018.18

Artículo de investigación. Fecha de recepción: 15/06/2018. Fecha de aceptación: 27/11/2018

\author{
Dilia Ahumada-Rojas; Edinson Villa-Castañeda; Lisbeth Taborda-Cotes; \\ Martha Cabarcas-Matta; Meira Sierra-Rodríguez; Abel Polo-Domínguez; \\ Nely Vides-Pertuz; Alicia Barraza-Sierra; Christian Rivera-Acosta; \\ Gustavo Vélez-García; Martha Vivero-Vargas; Edwin Angarita-Jaimes; \\ María Hernández-Maldonado; Zamir Montero-Pertuz; \\ Caterine Lizarazo-Barrera y Luis Alemán-Escobar1 \\ IED John f Kennedy (Colombia)
}

Para citar este artículo:

Ahumada-Rojas, D., Villa-Castañeda, E., Taborda-Cotes, L., Cabarcas-Matta, M., Sierra-Rodríguez, M., Polo-Domínguez, A., VidesPertuz, N., Barraza-Sierra, A., Rivera-Acosta, C., Vélez-García, G., Vivero-Vargas, M., Angarita-Jaimes, E., Hernández-Maldonado, M., Montero-Pertuz, Z., Lizarazo-Barrera, C. y Alemán-Escobar, L. (2018). Comprensión lectora mediante la investigación como estrategia pedagógica apoyada en TIC. Cultura. Educación y Sociedad 9(3), 153-160. DOI: http://dx.doi.org/10.17981/cultedusoc.9.3.2018.18

\section{Resumen}

En la actualidad, los sistemas educativos buscan diversas estrategias para fortalecer los procesos de lectura, basándose en las nuevas tecnologías de la información y comunicación como medio de motivación que genera aprendizaje y dinamiza las practicas pedagógicas de los docentes. El estudio tuvo como propósito fortalecer la comprensión lectora mediante la investigación como estrategia pedagógica apoyada en las tecnologías de la información y comunicación TIC. Este estuvo guiado bajo los lineamientos metodológicos de la IEP con un diseño basado en las trayectorias de indagación. Se utilizaron como técnicas de recolección de información la observación participante y el diario de campo. La población estuvo conformada por cuarenta (40) estudiantes de básica primaria con edades comprendidas entre los ocho (8) y doce (12) años de la IED John F. Kennedy del municipio de Fundación- Magdalena. Se evidencio un avance en la comprensión lectora mediante la investigación y las herramientas virtuales observando mayor interés y desempeño en el aula de clase mediante estas estrategias.

Palabras clave: comprensión lectora, investigación como estrategia pedagógica, tecnologías de la información y la comunicación.

\section{Abstract}

At present, educational systems seek different strategies to strengthen reading processes, based on new information and communication technologies as a means of motivation that generates learning and dynamizes the pedagogical practices of teachers. The purpose of the study was to strengthen reading comprehension through research as a pedagogical strategy supported by ICT information and communication technologies. This was guided under the methodological guidelines of the IEP with a design based on the trajectories of inquiry. The participant observation and the field diary were used as data collection techniques. The population consisted of forty (40) elementary school students aged between eight (8) and twelve (12) years of the IED John F. Kennedy of the municipality of Fundación-Magdalena. There was evidence of an advance in reading comprehension through research and virtual tools, observing greater interest and performance in the classroom through these strategies

Keywords: reading comprehension, research as a pedagogical strategy, information and communication technologies. 


\section{Introducción}

Los docentes investigadores de la IED John F. Kennedy identificaron debilidades en los estudiantes para interpretar la información en diferentes ámbitos como lectura crítica, problemas matemáticos, razonamiento lógico y otros, dicha problemática se evidencia en los bajos resultados en las pruebas saber 2016 publicadas en 2017, bajos resultados en las notas, desinterés a algunos contenidos propios de cada área, estas deficiencias se pueden identificar gracias a las continuas observaciones y vivencias en el salón de clases, en las horas de descanso, en las salidas pedagógicas, consejos académicos y comités de promoción y evaluación en las cuales os docentes registran y comentan las dificultades de aprendizaje de sus estudiantes en las diferentes áreas.

Dicha situación está estancando el desarrollo de la calidad educativa ya que la institución estipula en su proyecto educativo institucional brindar una educación integral para el liderazgo, autonomía, participación, construcción participativa de comunidad educativa, calidad académica e investigativa, plan de estudios basado en proyectos pedagógicos multidisciplinarios, procesos académicos virtuales (Redes de información), fomento de la creatividad y la expresión artística y cultural, asesores externos de reconocida trayectoria administrativa, docente, didáctica y pedagógica. Buenos materiales didácticos que permiten el desarrollo cognitivo, y recursos audiovisuales. por ello el grupo Lectotic ha diseñado una propuesta enmarcada en cómo mejorar la comprensión lectora a través de la IEP basada en TIC.

Por otra parte, de acuerdo al Portal Colombia Aprende del MEN (Ministerio de Educación Nacional) el Índice Sintético de Calidad Educativa-(ISCE) nacional es de 5.61 y la entidad territorial certificada el departamento, Magdalena es de 4.49. de esta manera la formación académica en básica secundaria y media en lenguaje a nivel nacional está por debajo de los índices que exigen el Ministerio de Educación Nacional.

Para el caso de la Institución Municipal John F. Kennedy de Fundación departamento del Magdalena, se encuentra en la escala de 1 a 10, esta tiene un puntaje de 3,71 está por debajo del índice nacional que es de 5,61 y por debajo de la ETC que es de 4,49. Además, el ISCE, la institución educativa John F. Kennedy, en los procesos de desempeños que evalúan en las pruebas saber, presenta un bajo promedio según los resultados obtenidos, de esta forma, el índice sintética de la institución educativa en secundaria es: Progreso 0,01 Desempeño 2,06, Eficiencia 0,88 Ambiente Escolar 0,75 = 3.71; Estos resultados evidencias el bajo rendimiento académico de la población estudiada Con la apreciación de estos promedios en los diferentes componentes evaluados, se puede evidenciar que los estudiantes del ciclo secundaria, son promovidos con unas falencias o una serie de problemas que generan en ellos un bajo rendimiento.

Dentro del maco de proyecto fortalecimiento de la cultura ciudadana y democrática en $\mathrm{CT}+\mathrm{I}$ a través de la IEP apoyada en TIC en el departamento del Magdalena programa CICLON, se determina un espacio para maestros (as) participantes en el proceso de formación para que desarrollen proyectos de investigación que apunten a resolver problemas del contexto, como lo es el caso del grupo investigativo Iectotic integrado por los docentes investigadores de la IED John F. Kennedy los cuales han identificado debilidades en los estudiantes para interpretar la información en diferentes ámbitos como lectura crítica , problemas matemáticos, razonamiento lógico y otros, dicha problemática se evidencia en los bajos resultados en las pruebas saber 2016. 
Comprensión lectora mediante

la IEP apoyada en TIC

Se entiende por competencias lectoras saber leer, interpretar y criticar un texto. A lo que Giroux y Tremblay (2004), da un sentido amplio al concepto de comprensión lectora al considerar que es la interpretación que se produce del texto dentro de un texto y que en la crítica se construye un texto en contra de un texto. El interpretar en cierto modo significa una capacidad para desgarrar posiciones ocultas en medio de una estructura de sistemas de valores culturales cotidianos.

Es por ello, que las palabras en nuestro mundo social, contempla una competencia donde el estudiantado poner en acción una serie de estrategias y habilidades tendientes a interpretar el mensaje implícito del texto, valorarlo para asumir una posición frente a lo que plantea el autor y hacer uso de una información para construir un nuevo conocimiento. Este debe ser el objetivo del acercamiento que hace el docente en todos los niveles académicos para que sus estudiantes puedan, a través del texto, establecer un diálogo con el texto y así, capturar el mensaje de su autor (Marciales, 2003).

Por otro lado, Teberosky, (2002). Concibe la lectura como un medio a través del cual el ser humano procesa de manera sistematizada la información recibida a través de códigos gráficos, integrando otros procesos como la comprensión y el análisis de la información, del mismo modo señala, que, a pesar de los grandes avances y las creaciones realizadas por el hombre, el proceso de lectura le permitirá comprender e interpretar la ciencia para continuar avanzado.

Según González, (2013). La lectura es un proceso que debe ser estimulado desde la infancia por padres y profesores, desde la escuela los docentes deben emplear estrategias pedagógicas para crear un hábito de lectura en sus estudiantes, formándolos competencias lingüísticas para que posteriormente puedan aportar nuevo conocimiento, originando así múltiples transformaciones en la producción de saber.

$\mathrm{Al}$ respecto Ferreiro (2005) manifiesta, que el ser humano debe adquirir la competencia de lectura crítica frente los textos que desarrolle, donde el sujeto le encuentre sentido y coherencia a los contenidos plasmados por el autor en su escrito, por tal razón dicha competencia está ligada a la comprensión lectora, la lectura es una actividad que permite analizar, decodificar e identificar lo que otra persona quiere decir, sin embargo, hay que tener en cuenta que la lectura no solo implica decodificar signos gráficos, sino buscarle un sentido al texto y transformar o nutrir los conocimientos previos a partir de la interiorización del texto.

Las instituciones educativas a diario utilizan textos básicos que orientan al estudiante en los contenidos programáticos que se van imparten en el año escolar; no obstante, la mayoría de los estudiantes hacen una corta revisión de los libros, puesto que en su mayoría perciben la lectura como una pérdida de tiempo. Además, no dedican tiempo a la búsqueda de nuevos textos que sirvan de apoyo en su formación; por lo que, a pesar de tratar con materiales impresos cotidianamente, no leen habitualmente (Lasso, 2013).

Siguiendo los lineamientos pedagógicos del Programa Ciclón, la estrategia de autoformación, formación de saber y conocimiento y apropiación para maestros(as) acompañantes investigadores y coinvestigadores en los lineamientos del programa de la IEP apoyada en TIC, se asume como un espacio de producción de saber y conocimiento de los maestros(as) beneficiados por el programa CICLÓN, a partir de la reflexión de la práctica pedagógica; reflexión que invita a considerar el desarrollar un proceso de investigación y sistematización de esta, que permita introducir en el currículo pautas y medidas que posicionen 
la investigación como un eje transversal y como estrategia pedagógica apoya en las TIC; viabilizando esto el desarrollo y fortalecimiento de las capacidades, habilidades, competencias tecnológicas, científicas, comunicativas, pedagógicas y de gestión curricular que deben estar presentes en el perfil docente.

Es necesario recalcar que, la investigación como estrategia pedagógica ejerce un impacto vinculante entre la práctica pedagógica y la investigación, se le brinda a las estudiantes la posibilidad de apropiarse de la lógica del conocimiento y de herramientas propias de la ciencia con la mirada puesta en la sociedad y sus necesidades, proyectándose al aprendizaje significativo que se basa en atribuir significado al material objeto de aprendizaje, no sólo se deben actualizar sus esquemas de conocimiento sino también revisar, modificar y enriquecer este conocimiento (Ausubel, 2001).

Por otro lado, la innovación, la interacción y su justificación, constituye una estructura de un conocimiento escolar donde se interrelacionan cuatros componentes; de los cuales intervienen el mundo de los estudiantes, los fines de la escuela, la ciencia para el contexto y el método para lograr un resultado idóneo (Duque, 2017). De igual manera, el maestro como mediador es el responsable de llevar a los estudiantes a un entorno investigativo-científico, donde se busca la consolidación en la comprensión de un lenguaje científico. La concepción que tiene el maestro de la ciencia ligada a la pedagogía genera campos de saber, esto conlleva a un cambio en la idea del maestro y su rol. Schon (1991), lo reconoce más como productor de saber que como implementador de procesos prediseñados, los denomina como profesionales reflexivos.

Es necesario abordar la importancia de la investigación como una estrategia idónea a partir de un viraje general de las variables que se van a dando en un momento determinado. Por su parte, el concepto de educación, trae implícito el manejo de competencias, por lo cual hoy en día la educación se concibe como un fenómeno dinámico constructivo y con iniciativas innovadoras en la educación actual Imbernon (2007), además de facilitar el acceso a una formación basada en conocimientos, permite el desarrollo de habilidades necesarias en la sociedad de la información. En la actualidad, la educación contempla mejorar sus prácticas fomentando el uso de los medios electrónicos.

Es necesario que el asumir la investigación puede conllevar a procesos pedagógicos que requiere un entorno contextual que implique una formación pertinente a establecer estrategias investigativas para construir una apropiación basadas en lecturas que creen y den soluciones a problemas del conocimiento en la ciencia, la tecnología y la innovación en las particularidades de un mundo en el cual los procesos tecnológicos en marcha signifiquen también nuestras realidades y la profundización de la desigualdad. Es importante recordar a manera de ejemplo cómo hay más redes telefónicas e internet en la isla de Manhattan que en toda África. Es decir, plantear trabajar con la investigación exigía sacarla del marco optimista de que es la creadora de este nuevo mundo y una necesidad universal y escrutar qué significaba eso para hacerlo real en las particularidades de una región como la nuestra (Mejía y Manjarres, 2011).

En efecto, se trata de tener la certeza que el conocimiento pertinente a las tecnologías, mediante procesos investigativos conllevan a encontramos frente a productos socialmente construidos, y cuando nosotros los convertimos en procesos educativos estamos haciendo de ellos una interacción construida con nuevas mediaciones sociales, la cual permite hacer frente a una necesidad de reconfiguraciones pedagógicas debidas a las transformaciones en el conocimiento y la tecnología, sino la necesidad de releerlas a todas como un asunto muy importante en la reconstrucción de lo público 
y lo político en estos tiempos en los cuales, como "hay proyecciones, pero no proyectos" (Lechner, 2002).

En ese sentido, el esfuerzo por construir una propuesta pedagógica que retomara la investigación en estos contextos se enclava en un debate ético-político sobre la asignación de esos bienes determinantes en la constitución de desigualdades, y le apuesta por construir desde la más tierna infancia capacidad y autonomía para preguntarse y, desde el ejercicio práctico de la investigación, impugnar esa asignación constructora de injusticia en la esfera de la individuación, la socialización, la nación y el escenario internacional (Mejía y Manjarres, 2011).

Por su parte la integración de las TIC al contexto educativo es un proceso que ha incrementado a nivel mundial, considerándose una era de alfabetización digital, sin embargo, su incorporación no se limita a contar con las herramientas tecnológicas, como equipos y programas, sino a la construcción de estrategias educativas que propicien ambientes de aprendizaje (Díaz, 2013).

Las TIC facilitan el acceso a la información, sin embargo, no todo lo que se obtiene mediante las TIC es conocimiento estricto, se hace necesario realizar una serie de estrategias para que el sujeto desarrolle la capacidad de identificar información científica que le permita interpretar y construir su propio conocimiento a través de la revisión teórica. Lo cual se convierte en un reto debido a que la educación tradicional ha priorizado en la memorización y la repetición de frases establecidas. En el caso de las TIC se expresa en actividades de copiar y pegar información, donde se hace necesario redireccionar al estudiante para que tome la información como referente, le encuentre un sentido y significado y la haga propia, fortaleciendo así el proceso de lectura, comprensión e interpretación de textos y escritura (Díaz, 2013).

\section{Metodología}

La metodología del proyecto de investigación se realizó a partir de los recorridos de las trayectorias de indagación, basados en la integración de la IEP apoyada en TIC al aula, articulado al plan de estudios, desde las diferentes áreas se estructuraron temáticas relacionadas con la robótica educativa, la finalidad de este proceso investigativo, fue generar una trasformación social y educativa a través del aprendizaje en contexto, involucrando intereses, motivaciones y realidades de los estudiantes para generar conocimiento científico, donde se establecieron siete (7) momentos que especifican en la ejecución y las metas trazadas para darle solución a la pregunta problema planteada. A continuación, se presentan los diferentes momentos.

Momento 1. En conjunto, los docentes investigadores diseñan el plan de acción que incluye la selección de los temas y las estrategias investigativas correspondientes; según sus áreas académicas se seleccionan las temáticas, respondiendo a tres criterios: a) áreas cuánticas, complejidad, b) áreas de humanidades, temas extensos y donde los estudiantes han demostrado bajo rendimiento y c) áreas tecnológicas, temas de interés para los estudiantes. Asimismo, se diseñan instrumentos de diagnóstico y evaluación.

Momento 2. Los docentes a través del instrumento de diagnóstico, diseñado según el modelo de las pruebas estandarizadas que identifican el nivel de comprensión lectora de la población. Con análisis descriptivo.

Momento 3. Se despliega la implementación y transversalización de las estrategias investigativas dirigidas a los estudiantes y orientadas por los docentes. 
Momento 4. Desde el área de tecnología e informática se desarrolla la formación en la creación de objetos virtuales de aprendizaje, apropiación desde la praxis basándose en temas de interés y posteriormente elaborados y consolidados como resultado de la indagación de los temas señalados por las áreas específicas.

Momento 5. Aplicación del instrumento de evaluación, diseñado según el modelo de las pruebas estandarizadas que identifican el nivel de comprensión lectora de la población. Análisis descriptivo.

Momento 6. Análisis e interpretación de resultados

Momento 7. Reflexión sobre el impacto del currículo al incluir a IEP en la práctica pedagógica.

Empleando también las trayectorias de indagación del docente que construyen el saber y las estrategias desde el trabajo colaborativo y la indagación, teniendo en cuenta las características del grupo humano beneficiado y la comunidad. Todo a partir de la implementación de las estrategias planteadas que responden a las características y realidades del grupo, la comunidad y las herramientas accesibles de la institución. Se utilizó como instrumento de recolección de información la bitácora que, descrita desde cada momento, se registran los acontecimientos según el criterio de referencia o los emergentes en cada una de ellas.

Por tanto, los estudiantes recorrerán su propia trayectoria de indagación orientado por el docente que les permitirá utilizar instrumentos como: encuestas, guías de Observación, consultas en fuentes especializadas virtuales, entrevistas, análisis documental y de contenido de fuentes oficiales (censos - ficheros), y las historias de vida para recuperar la información de la comunidad, en especial de los ancianos o a partir de fichas bibliográficas para registrar el material impreso y virtual producido tanto video-gráficas e infografías.

\section{Resultados}

Según Mejía y Manjarres (2011) en la misma dinámica, la investigación como estrategia pedagógica está dirigida con la posibilidad de comprender una transformaciones enfocadas en un ejercicio que realizan los grupos investigativos para producir cambios necesarios en la práctica pedagógica, e inician en ese entorno no por una ley o un mandato, sino porque encuentran allí que pueden ser educadores de otra manera, sin miedo, en búsqueda, sin certezas, pero con la compañía del grupo con el cual van reelaborando su práctica, al maestro y la maestra que trabajan con la IEP para consolidar el ejerció en la práctica que garanticen tales actividades pertinente a una enseñanza cónsonas con el entorno estudiantil.

Dadas las condiciones que anteceden, es necesario construir una cultura ciudadana y democrática en ciencia, tecnología e innovación para estos tiempos en un mundo construido sobre el conocimiento a una propuesta en referencia al conocimiento científico, mediante tecnologías de la información fundamentadas bajos criterios de una idea de justicia educativa y justicia curricular, trabajando por construir sociedades más justas y menos desiguales, con un sentido que involucre a los estudiantes jóvenes a los procesos investigativos como parte de la búsqueda iniciada desde la propuesta metodológica que contiene como valores fundamentales esas capacidades del ser humano, como son la parte (cognitivas, afectivas, valorativas y de acción) sobre las cuales se fundamenta la investigación como estrategia pedagógica).

A partir de estos objetivos expuestos se logra mejorar considerablemente la comprensión lectora mediante la IEP apoyada en la creación de objetos virtuales de aprendizaje. Todo esto después de evaluar el nivel de comprensión lectora a partir del diseño de una ruta de implementación de la IEP para la comprensión lectora, la cual 
diagnostica el nivel de compresión lectora e implementa la IEP desde las áreas académicas. Formando a los estudiantes desde el área de informática en la elaboración de objetos virtuales de aprendizaje desde las diversas áreas académicas.

\section{Conclusiones}

Después de haber llevado a los objetos virtuales de aprendizaje a través de la IEP como elemento propiciador de la práctica educativa se considera que los practicantes inmersos en esta metodología evidencian una toma de conciencia reflexión y apropiación de esta concepción educativa y de la manera como ella está presente en sus teorías, metodologías, interacciones, mediaciones, campos o espacios de aprendizaje en la comprensión de los textos que abordaron durante este proceso.

Por otra parte, se considera que gracias a la inclusión de la IEP en el currículo y la promoción de las competencias investigativas en los estudiantes beneficiados con el proyecto "mejoramiento de la comprensión lectora a través de la IEP basada en la creación de objetos virtuales de aprendizaje" se ha logrado aportar un importante grano de arena en la construcción de una cultura ciudadana y democrática en un mundo en el cual el conocimiento y la tecnología son fundamentos de nuevas desigualdades además de lograr un aprendizaje resultante de dinámicas de personas y grupos que impulsan esta nueva propuesta donde se aprende a investigar solo investigando.

A pesar de no haber llevado la totalidad de esta nueva forma de educar, se logra consolidar las bases para que la IED John f Kennedy logre adoptar la IEP año tras año, ya que a través de la misma se pueden resolver problemas educativos como lo es esta propuesta la cual busca mejorar la comprensión lectora. Y de conseguirlo seguramente se podrá decir que se ha dado un gran salto en calidad educativa

\section{Referencias}

Argudín, Y. (2005). Educación basada en competencias. México, D.F.: Trillas.

Ausubel, D. (2001). Adquisición y retención del conocimiento. Madrid: Paidós.

Duque, I. (22 de abril, 2017). La investigación como estrategia pedagógica en el aula [Mensaje en un blog]. Recuperado de https://compartirpalabramaes$\mathrm{t} \mathrm{r}$ a . o r g / c o l u m n a s / la-investigacion-como-estrategia-pedagogica-en-el-aula

Giroux, S. y Tremblay, G. (2004). Metodología de las Ciencias Humanas. La investigación en acción. México, D.F.: Fondo de cultura económica.

Hodgins, W. (1992). Evaluating the correspondence of educational software to learning theories. Recuperado de https://dl.acm.org/citation.cfm?id=249 1845.2491882\&coll=DL\&dl=ACM

Imbernón, F. (2007). La formación y el desarrollo profesional del profesorado. Hacia una nueva cultura profesional. Barcelona: Graó.

Institución Tecnológica Colegio Mayor de Bolívar. (2013). Proyecto estratégico de fomento a la permanencia PEFPE: objetos virtuales de aprendizaje. Recuperado de http://www.colombiaaprende. edu.co/html/micrositios/1752/articles-345057_recurso_2.pdf

Jiménez, J. y Salas, M. (2017). Aplicación de modelos econométricos para estimar la aceptabilidad de una tasa por congestión vehicular. INGE CUC, 13(2), 60-78. https://doi.org/10.17981/ ingecuc.13.2.2017.08

González, D. (2013). Importancia de los paratextos en la lectura e Interpretación de Textos Literarios. Multiciencias. 13(2). 180-189. Disponible en http://www.redalyc.org/articulo. oa?id=90428841010 
Lasso, R. (2013). Importancia de la lectura. Recuperado de http://bivir.uacj.mx/ LibrosElectronicosLibres/UACJ/Importancia_de_la_lectura.pdf

Lechner, N. (2002). Las sombras del mañana. La dimensión subjetiva de la política. Santiago de Chile: LOM.

Marciales, G. (2003). Pensamiento crítico: diferencias en estudiantes universitarios en el tipo de creencias, estrategias e inferencias en la lectura crítica de textos. (Tesis). Universidad Complutense de Madrid, Madrid. Recuperado de http://biblioteca.ucm.es/tesis/edu/ ucm-t26704.pdf

Mejía, M. y Manjarres, M. (2011). La investigación como estrategia pedagógica una apuesta por construir pedagogías críticas en el siglo XXI. Praxis \& Saber, 2(4), 127-177.
República de Colombia. Ministerio de Educación Nacional. (2017). Objetos virtuales de aprendizaje e informativos. [Documento en línea]. Recuperado de http://www.colombiaaprende.edu. co/html/directivos/1598/article-172369.html

Rojas, C. (2015). Objetos virtuales de aprendizaje como herramienta para la enseñanza del álgebra en el grado octavo de la Institución Educativa Ana de Castrillón. (Tesis). Universidad. Nacional de Colombia, Medellín.

Schon, D. (1991). La formación de profesionales reflexivos. Hacia un nuevo diseño de la enseñanza y el aprendizaje en las profesiones. Madrid: Paidós. 